\title{
Impacto da saúde bucal de pacientes com paralisia cerebral no cuidador
}

- Vanessa Gonçalves Moreira Faculdade de Odontologia, Universidade Tiradentes, Aracaju, SE, Brasil

- Helare Adane Andrade Santos Faculdade de Odontologia, Universidade Tiradentes, Aracaju, SE, Brasil

- Paulo RicardoSaquete MartinsFilho Faculdade de Odontologia, Universidade Federal deSergipe, Lagarto, SE, Brasil

- Alina Lúcia de Oliveira Barros Hospital Universitário, Universidade Federal de Sergipe, Aracaju, SE, Brasil

- Sara Juliana de Abreu de Vasconcellos Faculdade de Odontologia, Universidade Tiradentes, Aracaju, SE, Brasil

RESUMO | Objetivo: O objetivo deste estudo foi avaliar o impacto da saúde bucal das crianças e adolescentes com paralisia cerebral (PC) na vida dos seus cuidadores. Materiais e Métodos: Trata-se de um estudo transversal e de abordagem quantitativa, realizado em Aracaju/SE, Brasil, de julho de 2019 a abril de 2020. Os dados de 22 cuidadores foram coletados por meio dos questionários sócio demográfico e Family Impact Scale (FIS) na Unidade Odontológica para Pacientes Especiais da Universidade Federal de Sergipe (Hospital Universitário). Resultados: Observou-se a prevalência de mulheres como cuidadoras principais (81\%), e dos 22 dos entrevistados, 19 se dedicavam em tempo integral. Com relação ao perfil do paciente com PC, $67 \%$ eram do sexo masculino, entre 11 e 15 anos (38\%). Para a análise do FIS, foram encontrados médias de impacto de 33 pontos. Conclusão: Foi observado alto impacto da saúde bucal dos pacientes com PC em seus cuidadores. Em contrapartida, foi observado que os cuidadores estão buscando cada vez mais recursos que possibilitem o bem-estar dos seus filhos, não só físico, como bucal, por meio de participação em programas governamentais que sejam voltados para a educação em saúde bucal.

DESCRITORES | Saúde bucal; Cuidadores; Paralisia Cerebral.

ABSTRACT | Impact of oral health of patients with cerebral palsy in the caregiver - Objective: Our study sought to evaluate how oral health of children and adolescents with CP affects their caregivers' life. Materials and Methods: This is a cross-sectional study with a quantitative approach conducted in Aracaju, state of Sergipe, Brazil, from July 2019 to April 2020. Data from 22 caregivers were collected at the Dental Unit for Special Patients at the Universidade Federal do Sergipe (Hospital University), using a sociodemographic questionnaire and the Family Impact Scale (FIS). Results: Women were more prevalent as main caregivers (81\%), and 19 out of the 22 interviewees were full time caregivers. Regarding the profile of the patient with $\mathrm{CP}, 67 \%$ were boys between 11 and 15 years old (38\%). For the analysis of the FIS, an impact average of 33 points were found. Conclusion: We observed a high impact of oral health of patients with CP on their caregivers. On the other hand, caregivers are increasingly looking for resources that enable not only their children's physical, but also their oral well-being, with participation in government programs aimed at education in oral health.

DESCRIPTORS | Oral Health; Caregivers; Cerebral Palsy.

AUTOR CORRESPONDENTE | • Vanessa Gonçalves Moreira Faculdade de Odontologia. Universidade Tiradentes. • Rua Siriri, 263 Aracaju, SE, Brasil • 49010-450 • E-mail: vanessagoncaalves@outlook.com

- Recebido 30 Julho, 2020 • Aceito 26 Outubro, 2020

- Dol: http://dx.doi.org/10.11606/issn.2357-8041.clrd.2021.173063 


\section{INTRODUÇÃO}

A paralisia cerebral (PC) é definida como uma lesão crônica permanente e não progressiva do sistema nervoso. Pode ser considerada uma doença multifatorial, de etiologias pré-natais, perinatais e/ou pós-natal e acomete o desenvolvimento biológico, desde os tônus, o reflexo, a postura, até o desenvolvimento motor do indivíduo. ${ }^{1} \mathrm{~A}$ gravidade da $\mathrm{PC}$ está vinculada à escassez da função motora junto à dificuldade da criança em realizar afazeres básicos do seu cotidiano. A PC não tem cura, e é necessário administrar os sintomas para que haja um tratamento eficaz, minimizando a deformidade musculoesquelética. ${ }^{2,3}$

Os pacientes com PC carecem de uma higiene bucal satisfatória, e isso reflete em uma alta incidência de problemas frequentes de saúde bucal, dando ênfase para a cárie dentária e doenças periodontais. ${ }^{4} \mathrm{~A}$ cárie é resultado de um processo crônico que envolve fatores etiológicos como dieta, tempo, dente suscetível e micro-organismo. A suscetibilidade dessa doença envolve aspectos de hereditariedade e imunológicos, além de fatores intrínsecos como o fluxo e capacidade tampão da saliva. Ademais, outros problemas podem estar associados à qualidade bucal desses pacientes, como anormalidade na oclusão, bruxismo, efeitos colaterais dos medicamentos e dificuldade de acesso aos serviços odontológicos. ${ }^{5}$

A definição de "cuidador" pode ser considerada a partir de uma multiplicidade de conceitos. Segundo o Guia Prático do Cuidador, desenvolvido pelo Ministério da Saúde, esse conceito envolve qualidades como o amor, a solidariedade e a doação à humanidade. ${ }^{6}$ Um cuidador informal presta assistência não remunerada a alguém com quem tem um relacionamento pessoal, podendo abranger mães, pais, tios, avós etc.; além disso, o papel do cuidador agrega sobrecarga às atuações previamente existentes. O cuidador prioriza as necessidades do seu parentesco, e esse cenário gera uma pressão, devido às necessidades imediatas do doente; dessa maneira, esquece de si mesmo, podendo repercutir assim nas suas atividades diárias. 7

A saúde bucal é um componente da saúde geral e, quando comprometida, impacta negativamente a qualidade de vida do indivíduo. Os pais ou responsáveis pelas crianças com deficiência carecem de instruções na prevenção das doenças bucais, sendo imprescindível sua motivação para a adequada execução da higiene bucal, visto que, em muitos casos, a rotina está associada à dieta cariogênica, falta de hábitos de higiene e dificuldade de acesso a serviços odontológicos. Consequentemente, o alto empenho do cuidador e dedicação de horas no cuidado do ente parentesco afeta diretamente o seu tempo e empenho na ocupação pessoal, incluído bons hábitos de higiene oral. ${ }^{4}$

Nesse contexto, o acompanhamento clínico e os tratamentos dessa doença tornam o apoio familiar imprescindível para o paciente durante toda a vida, o que influencia a sobrecarga emocional vivida pelos cuidadores. ${ }^{8}$ Diante da importância dessa temática para a sociedade e para os profissionais de saúde, este estudo teve como objetivo avaliar o impacto da saúde bucal de pacientes portadores de PC e o que isso acarreta para o seu cuidador.

\section{MATERIAIS E MÉTODOS}

Trata-se de um estudo transversal e de abordagem descritiva e quantitativa. Os dados foram coletados durante o período de julho de 2019 a abril de 2020 na Unidade Odontológica para Pacientes com Necessidades Especiais (UDOPE), no Hospital Universitário (HU) da Universidade Federal de Sergipe (UFS), na cidade de Aracaju (SE), Brasil.

Foram entrevistados 22 cuidadores primários de pacientes com PC, por conveniência, incluindo cuidador primário independente do sexo e com no mínimo 18 anos de idade, que exerça a função há, no mínimo, doze meses e sem auxílio de remuneração. Foram excluídos cuidadores primários com deficiências motoras, sensoriais 
e/ou cognitivas que possam prejudicar a aplicação dos questionários (compreender e responder); além disso, também foram excluídos aqueles com dois ou mais pacientes com PC e os que se recusaram a participar da pesquisa.

Os cuidadores foram submetidos a uma entrevista individual, realizada em uma única etapa, sendo aplicado o Questionário Sociodemográfico do Cuidador Primário e Perfil do paciente com PC. Para os cuidadores, foram avaliadas variáveis como escolaridade, sexo, ocupação atual, renda familiar e quantidade de filhos. Quanto aos pacientes com PC, foram levantados dados sobre sexo, idade, presença de convulsões e auxílio benefício como o benefício de prestação continuada (BPC).

Também foi aplicado o Questionário para Avaliação do Impacto da Saúde Bucal no Familiar: Family Impact Scale (FIS). Essa escala avalia os efeitos que a condição bucal da criança apresenta na vida dos seus pais ou membros da família. O questionário é composto por 14 itens divididos em quatro subescalas: atividades dos pais e familiares, emoções dos pais, conflito familiar e dificuldade financeira. ${ }^{9}$ As respostas dos domínios variam de o a 4: "nunca - 1" a "quase todos os dias - 4". As pontuações são calculadas pela soma de todos os itens, podendo o final variar de o a 56. Assim, quanto maior a pontuação obtida, maior o grau de dificuldade enfrentada pelo cuidador, tendo em vista o maior impacto na sua qualidade de vida.

Para análise dos dados foi utilizado o Windows 365 (Excel 2010). As variáveis quantitativas foram expressas em valores absolutos e relativos. Os valores dos escores de Impacto da Saúde bucal na Qualidade de Vida foram expressos em média e analisados com ponto de corte para o impacto gerado no cuidador acima de 50\% dos pontos dos escores. Estudo aprovado pelo Comitê de Ética em Pesquisa com seres humanos do Hospital Universitário (HU) da Universidade Federal de Sergipe (UFS), seguindo as normas da resolução 446/2012 do
Conselho Nacional de Saúde (CNS), sob parecer $\mathrm{n}^{\mathrm{0}} 36970414 \cdot 4 \cdot 0000.5546$.

\section{RESULTADOS}

Participaram desta pesquisa 22 cuidadores informais assistidos na UDOPE, dos quais um cuidador foi excluído por não preencher corretamente o questionário, restando 21 cuidadores avaliados. Com relação ao perfil do cuidador informal, verificou-se que a maioria dos cuidadores era do sexo feminino (81\%), com idade entre 41 a 50 anos (33\%), estado civil casados (38\%), apresentando o vínculo de maior presença da mãe (76\%). Quanto ao grau de escolaridade, a maioria possuía ensino fundamental incompleto (52\%), ocupando o cargo de desempregado (65\%); além disso, a renda mensal era correspondente ao auxílio benefício do paciente com PC (39\%) (Tabela 1).

TABELA 1 || Perfil do cuidador a partir dos dados Sócio Demográfico.

\begin{tabular}{|c|c|c|c|c|c|}
\hline Sexo & $\mathrm{N}^{\circ}$ & $\%$ & Tempo de Dedicação & $\mathrm{N}^{\circ}$ & $\%$ \\
\hline Masculino & 4 & 19 & Parcial & 2 & 10 \\
\hline Feminino & 17 & 81 & Integral & 19 & 90 \\
\hline Idade & & & Renda do Cuidador & & \\
\hline De 20 A 30 & 5 & 24 & 01 Salário & 12 & 23 \\
\hline De 31 A 40 & 6 & 29 & 01 Salário + Auxílio & 12 & 23 \\
\hline De 41 A 50 & 7 & 33 & Sem Salário & 8 & 15 \\
\hline De 51 A 60 & 3 & 14 & Auxílio & 20 & 39 \\
\hline Vínculo & & & Ocupação Atual & & \\
\hline Pai & 2 & 9 & Autônomo & 6 & 30 \\
\hline Mãe & 16 & 76 & Do Lar & 1 & 5 \\
\hline $\begin{array}{l}\text { Irmão } \\
\text { Tia }\end{array}$ & $\begin{array}{l}2 \\
1\end{array}$ & $\begin{array}{c}10 \\
5\end{array}$ & $\begin{array}{l}\text { Desempregado } \\
\text { Trabalhador formal }\end{array}$ & $\begin{array}{c}13 \\
1\end{array}$ & $\begin{array}{c}65 \\
5\end{array}$ \\
\hline Grau de Escolaridade & & & Estado Civil & & \\
\hline $\begin{array}{l}\text { E. Superior Incompleto } \\
\text { E. Médio Completo }\end{array}$ & $\begin{array}{l}2 \\
7\end{array}$ & $\begin{array}{l}10 \\
33\end{array}$ & $\begin{array}{l}\text { Casado } \\
\text { União Estável }\end{array}$ & $\begin{array}{l}8 \\
5\end{array}$ & $\begin{array}{l}38 \\
24\end{array}$ \\
\hline $\begin{array}{l}\text { E. Fundamental } \\
\text { Incompleto }\end{array}$ & 11 & 52 & $\begin{array}{l}\text { Solteiro } \\
\text { Viúva }\end{array}$ & $\begin{array}{l}6 \\
2\end{array}$ & $\begin{array}{c}29 \\
9\end{array}$ \\
\hline Analfabeto & 1 & 5 & & & \\
\hline
\end{tabular}

Fonte: Pesquisa de campo.

Tendo em vista o perfil dos pacientes com PC, a amostra foi constituída de $67 \%$ dos pacientes do sexo masculino e os outros $33 \%$, do sexo feminino. A idade média dos pacientes era de 15 anos, e 12 pacientes apresentavam quadro convulsivo. 
Em relação ao benefício financeiro, 95\% dos pacientes recebiam BPC (Tabela 2).

TABELA 2 | Perfil dos pacientes com PC.

\begin{tabular}{l|c|c}
\hline Características Gerais & N & $\%$ \\
\hline Sexo & 7 & 33 \\
\hline Feminino & 14 & 67 \\
\hline Masculino & \multicolumn{2}{|c}{} \\
\hline Idade & 4 & 19 \\
\hline De 0 a 10 & 8 & 38 \\
\hline De 11 a 15 & 3 & 14 \\
\hline De 16 a 18 & 6 & 29 \\
\hline De 19 & & \\
\hline Benefícios & 20 & 95 \\
\hline Sim & 1 & 5 \\
\hline Não & & \\
\hline Convulsões & 12 & 57 \\
\hline Sim & 9 & 43 \\
\hline Não & &
\end{tabular}

Fonte: Pesquisa de campo.

O questionário FIS analisou como a saúde bucal do paciente com PC impactou a vida do seu cuidador nos últimos 3 meses. Foi encontrado maior valor no domínio Atividades dos Pais e Familiares (MP = 12,14), seguidos do domínio Emoções dos Pais ( $\mathrm{MP}=8,47)$, Conflito Familiar $(\mathrm{MP}=7,04)$ e Dificuldades Financeiras ( $\mathrm{MP}=2,57$ ) (Tabela 3).

TABELA 3 || Impacto de Saúde bucal do paciente com PC na vida do seu cuidador segundo os domínios (FIS).

\begin{tabular}{l|c}
\hline Domínios & Média Pontuação (MP) \\
\hline Atividades dos Pais Familiares & 12,14 \\
\hline Emoções dos Pais & 8,47 \\
\hline Conflito Familiar & 7,04 \\
\hline Dificuldades Financeiras & 2,57 \\
\hline Total & 30,22 \\
\hline
\end{tabular}

Fonte: Pesquisa de Campo.

\section{DISCUSSÃO}

Foi observado neste estudo maior prevalência do sexo feminino como cuidador primário principal de pacientes com PC, havendo maioria mãe e casada, constatação que pode estar relacionada ao apoio das mães em tempo integral a seus filhos em termos de alimentação, banho, vestimenta e locomoção. ${ }^{10,11}$ Verificou-se também que o nível de escolaridade pode estar associado ao entendimento a respeito do diagnóstico e, consequentemente, à forma dessas mães em encarar as dificuldades. É notório que o desconhecimento traz para esse grupo incertezas na forma de viver; portanto, pode-se afirmar que menores níveis de educação dos cuidadores dificultam também o entendimento, compreensão e aplicação dos conhecimentos sobre promoção de saúde bucal. ${ }^{12-15}$ Nesse estudo, 52\% dos entrevistados possuíam nível escolar fundamental incompleto.

Este estudo mostrou que 95\% dos pacientes com PC recebem auxílio financeiro, fazendo com que este seja a principal ou única renda familiar. Os cuidadores afirmam que, devido a dificuldades financeiras, o auxílio ajuda a conciliar as despesas relacionadas à compra de medicamentos de uso contínuo, transporte para consultas, alimentação adequada e recursos específicos que tragam o desenvolvimento de habilidades motoras para o paciente com PC. ${ }^{16} \mathrm{O}$ estudo feito por Assumpção e Kucznski com mães de crianças com doenças crônicas aponta que elas referiam estar satisfeitas frente à vida, em especial com os bens materiais que possuíam e que os seus filhos usufruíram, ${ }^{17}$ corroborando com o bem-estar material deles, mesmo nas famílias de menor poder aquisitivo, o que pode também ser confirmado nessa pesquisa onde o domínio Dificuldades Financeiras apresentou média pontuação de 2,57.

Estudos defendem que os cuidadores apresentam opiniões distintas em relação ao ato de cuidar, considerando a falta de apoio, o isolamento da família, além das características da criança, como gravidade da deficiência motora e intelectual e o meio social em que vive, sendo esses fatores contribuintes para o estresse e pressão psicológica. ${ }^{8,18}$ Isso pode estar vinculado à falta de acesso ao tratamento e à reabilitação para melhor prognóstico dessa deficiência devido às barreiras 
econômicas e sociais, o que afeta diretamente a dinâmica da família. ${ }^{19}$

Os resultados do questionário FIS apontaram uma variação dos escores geral de 19 a 47 pontos, com escore máximo desse questionário de 56 pontos, o que denota uma média de 33 pontos para essa amostra e média dos domínios de 30,22 pontos, significando um impacto relativo das condições bucais da criança na vida do cuidador. Evidências mostram que, devido ao atraso de desenvolvimento neuropsicomotor envolvendo a área sensorial e intelectual dos pacientes com PC, esses apresentam dificuldades em manter a saúde bucal ou comunicar suas necessidades de saúde bucal a seu cuidador. ${ }^{20,21}$ As preocupações gerais de saúde dessas crianças geralmente resultam na negligência de sua saúde oral e, dessa forma, esse aspecto não é considerado relevante para muitos cuidadores. A posteridade desses cuidados gera a gravidade e maior frequência dos distúrbios bucais em crianças com PC, o que pode reduzir a qualidade de vida dos cuidadores. Isso faz com que exista busca constante por serviços de saúde bucal, ${ }^{22}$ como foi visto nesta pesquisa, em que grande parte dos pacientes com PC estão em atendimento odontológico frequente.

\section{CONCLUSÃO}

O impacto no nível de saúde bucal dos pacientes com PC na vida dos seus cuidadores mostrou-se elevado. Mesmo com a busca constante por serviços odontológicos que contemplam esse grupo de pacientes, há necessidade de realizar atenção em saúde bucal: serviço assistencial curativo alicerçado em prevenção e promoção de saúde bucal para a família, com a melhoria das condições bucais dos pacientes com PC, diminuindo o impacto negativo da má saúde bucal do PC na vida de seus cuidadores.

\section{REFERÊNCIAS}

1. Lemos ACO, Katz CR. Condições de saúde bucal e acesso ao tratamento odontológico de pacientes com paralisia ce- rebral atendidos em um centro de referência do Nordeste Brasil. Rev CEFAC. 2012;14(5):861-71. doi: 10.1590/S151618462012005000045

2. Pereira HV. Paralisia cerebral. Resid. 2018;8(Supl 1):49-55. doi: 10.2506o/residpediatr

3. Wimalasundera N, Stevenson VL. Cerebral palsy. Practical Neurology. 2016;16(3):184-94. doi: 10.1136/ practneurol-2015-001184

4. Soares J, Volpato LER, Castro PHS, Lambert NA, Borges AH, CARVALHOSA AA. Avaliação do conhecimento sobre saúde bucal de pais e cuidadores de crianças e adolescentes com deficiência. J Health Sci Inst. 2013;31(3):239-43.

5. Pini DM, Fröhlich PCGR, Rigo L. Oral health evaluation in special needs individuals. Einstein. 2016;14(4):501-7. doi: 10.1590/s1679-45082016ao3712

6. Brasil. Guia Prático do Cuidador. 1 ed. Brasília: Ministério da Saúde; 2008. 64 p.

7. Fernandes CS, Angelo M. Family Caregivers: what dotheyneed? An integrative review. Rev Esc Enferm USP. 2016;50(4):672-8. doi: 10.1590/Soo80-623420160000500019

8. Keniş-Coşkun Ö, Atabay CE, Şekeroğlu A, Akdeniz E, Kasil B, Bozkurt G, et al. The relationship between caregiver burden and resilience and quality of life in a Turkish pediatric rehabilitation facility. J Pediatr Nurs. 2019;52:e108-e113. doi: 10.1016/j.pedn.2019.10.014

9. Goursand D, Paiva SM, Zarzar PM, Pordeus IA, Allison PJ. Family Impact Scale (FIS): psychometric properties of the Brazilian Portuguese language version. Eur J Paediatr Dent. 2009;10(3):141-6. PMID: 19761289

10. Chiluba BC, Moyo G. Caring for a cerebral palsy child: A caregivers perspective at the University Teaching Hospital, Zambia. BMC Res Notes. 2017;10(1):724. doi: 10.1186/s13104-017-3011-o

11. Albayrak I, Biber A, Çaliskan A, Levendoglu F. Assessment of pain, care burden, depression level, sleep quality, fatigue and quality of life in the mothers of children with cerebral palsy. J Child Health Care. 2019;23(3):483-94. doi: 10.1177/1367493519864751

12. Noronha DD, Martins AMEB, Dias DS, Silveira MF, De Paula $\mathrm{AMB}$, Haikal DSA. Qualidade de vida relacionada à saúde entre adultos e fatores associados: Um estudo de base populacional. Ciênc Saúde Coletiva. 2016;21(2):463-74. doi: 10.1590/1413-81232015212.01102015

13. Guerreiro PO, Garcias GL. Diagnóstico das condições de saúde bucal em portadores de paralisia cerebral do município 
de Pelotas, Rio Grande do Sul, Brasil. Ciênc Saúde Coletiva. 2009;14(5):1939-46. http://dx.doi.org/10.1590/S141381232009000500036

14. Silva ELMS, Goés PSA, Vasconcelos MMVB, Jamelli SR, Eickmann SH, Melo MMDC, et al. Cuidados em saúde bucal a crianças e adolescentes com paralisia cerebral: percepção de pais e cuidadores. Ciênc Saúde Coletiva. 2020;25(10):3773-84. doi: 10.1590/1413-812320202510.27972018

15. Cardoso AMR, Medeiros MMD, Gomes LN, Martins ML, Padilha WWN, Cavalcanti AL. Factors associated with health and oral health-related quality of life of children and adolescents with cerebral palsy. Spec Care in Dentist. 2018;38(4):216-26. doi: 10.1111/scd.12301

16. Vasconcelos VM, Frota MA, Pinheiro AKB, Gonçalves MLC. Percepção de mães acerca da qualidade de vida de crianças com paralisia cerebral. Rev Cogitare Enferm. 2010;15(2):238-44. doi: $10.5380 /$ ce.v15i2.17853

17. Assumpção FB Jr, Kucznski E. Qualidade de vida na infância e na adolescência: orientações para pediatras e profissionais da saúde mental. 1 ed. Porto Alegre: Artmed; 2010.
18. Tseng MH, Chen KL, Shieh JY, Lu L, Huang CY, Simeonsson RJ. Child characteristics, caregiver characteristics, and environmental factors affecting the quality of life of caregivers of children with cerebral palsy. Disabil Rehabil. 2016;38(24):2374-82. doi: 10.3109/09638288.2015.1129451

19. Glinac A, Matović L, Delalić A, Mešalić L. Quality of life in mothers of children with cerebral palsy. Acta Clin Croat. 2017;56(2):299-307. doi: 10.20471/acc.2017.56.02.14

20. Nqcobo C, Ralephenya T, Kolisa YM, Esan T, Yengopal V. Caregivers' perceptions of the oral-health-related quality of life of children with special needs in Johannesburg, South Africa. Health SA. 2019;24:1056. doi: 10.4102/hsag.v24io.1056

21. Lewis C, Vigo L, Novak L, Klein EK. Listening to Parents: A Qualitative Look at the Dental and Oral Care Experiences of Children with Autism Spectrum Disorder. Pediatr Dent. 2015;37(7):e98-104. PMID: 26883603

22. Camargos ACR, Lacerda TTB, Viana SO, Pinto LRA, Fonseca MLS. Avaliação da sobrecarga do cuidador de crianças com paralisia cerebral através da escala Burden Interview. Rev Bras Saúde Mater Infant. 2009;9(1). doi: 10.1590/S151938292009000100004 